\title{
Chaotic Tape Graphs
}

\author{
M. El-Ghoul \\ Mathematics Department, Faculty of Science \\ Tanta University, Tanta, Egypt \\ Tel: 104-684-812 E-mail: m.elghoul@msn.com \\ H. El-Zohny \\ Mathematics Department, Faculty of Science \\ Al-Azahar University, Cairo, Egypt \\ Tel: 168-308-166 E-mail: elzohny_7@yahoo.com \\ M. M. Khalil \\ Mathematics Department, Faculty of Science \\ Al-Azahar University, Cairo, Egypt \\ Tel: 106-518-009 E-mail: mona.khalil@msn.co
}

Received: December 15, 2010 Accepted: December 23, 2010 doi:10.5539/jmr.v3n2p182

\begin{abstract}
In this paper we will introduce new type of chaotic graphs, when vertices of these graphs are appearance like line and edges of these graphs are appearance like tape or ribbon and when these graphs carries physical characters. The representation of the chaotic graphs by matrices will be obtained. The transformations of the chaotic graph into itself and into another graphs will be discussed.
\end{abstract}

Keywords: Chaotic, Graphs

\section{Definitions and background}

Abstract graphs:

An abstract graphs $G$ is a diagram consisting of a finite non empty set of the elements, called "vertices" denoted by $V(G)$ together with a set of unordered pairs of these elements, called "edges" denoted by $E(G)$. The set of vertices of the graph $\mathrm{G}$ is called "the vertex - set of $G$ " and the list of edges is called "the edge - list of $G$ " (Gibbons A., 1985), (Giblin P.J., 1977).

Adjacency and incidence:

Let $v$ and $w$ be vertices of a graph. If $v$ and $w$ are joined by an edge $e$. then $v$ and $w$ are said to be adjacent. Moreover, $v$ and $w$ are said to be incident with $e$, and $e$ is said to be incident with $v$ and $w$ (Wilson R.J., 1972).

The adjacency matrix:

Let $G$ be a graph without loops, with $n$-vertices labeled $1,2,3, \ldots, n$. The adjacency matrix $A(G)$ is the $n \times n$ matrix in which the entry in row $i$ and column $j$ is the number of edges joining the vertices $i$ and $j$ (Wilson R.J.,1972).

The incidence matrix:

Let $G$ be a graph without loops, with $n$-vertices labeled $1,2,3, \ldots n$ and $m$ edges labeled $1,2,3, \ldots, m$. the incidence matrix $I(G)$ is the $n \times n$ matrix in which the entry in row $i$ and column $j$ is 1 if vertex $i$ is incident with edge $\mathrm{j}$ and 0 otherwise (Gross J.L.,Tucker T.W., 1987), (Wilson R.J., Watkins J.J., 1990).

Isometric folding:

Let $M$ and $N$ be smooth connected Riemannian manifolds of dimensions $m$ and $n$ respectively such that $m ; n$. A map $f$ : $M \rightarrow N$ is said to be an isometric folding of $M$ into $N$ iff for every piecewise geodesic path $\gamma: J \rightarrow M$ the induced path $f$ o $\gamma: J \rightarrow N$ is a piecewise geodesic and of the same length as $\gamma$ (Robertson S.A., 1977).

Folding and unfolding of graph:

(a) Let $f: G \rightarrow \bar{G}$ be a map between any two graphs $G$ and( not necessary to be simple) such that if $(u, v) \in G,(f(u), f$

$(v)) \in \bar{G}$. Then $f$ is called a "topological" of $G$ to provided that $d(f(u), f(v)<d(u, v)$ (Giblin P.J., 1977). 
(b) Let $g: G \rightarrow \bar{G}$ be a map between any two graphs $\mathrm{G}$ and (not necessary to be simple) such that if $(u, v) \in G$ ,$(g,(u), g(v)) \in \bar{G}$.Then $g$ is called a "topological unfolding" of to $\bar{G}$ provided that $d(g(u), g(v))>d(u, v)$ (El-Ghoul M., 2007).

\section{Retracts:}

A subset $A$ of a topological space $X$ is called a "retract" of $A$ if there exists a continuous map $r: X \rightarrow A$ (called a retraction) such that $r(a)=a \forall a \in A$, where $A$ is closed and $X$ is open. In other words, a retraction is a continuous map of a space onto a subspace leaving each point of the subspace fixed (El-Ghoul M.,El-Ahmady A., Rafat H., 2004).

Chaotic graphs:

A "chaotic" graph is a geometric graph that carries many physical characters (El-Ghoul M., 1993), (El-Ghoul M., ElAhmady A. and Homoda, 2006).

\section{Main Results}

In the following, we will define the chaotic graph as the graph which carries many physical characters. The representation of these chaotic graph by matrices will be defined. Also some transformations of this chaotic graph into itself will be acheived.

\subsection{Definitions}

The geometric tape graph $G$ is a diagram consisting of a finite non empty set of the elements with "line or curve" shape called "vertices" dented by $V(G)$ together with elements, with "tape" shape called "edges" denoted by $E(G)$. When that geometric tape graph carrying physical characters we called "chaotic tape graph" denoted by $G_{h}$. And there are two types of chaotic of tape graph:

a-Chaotic of one face: A physical characters carries on one face of geometric tape graph like painting, printing,glue ...etc.

b-Chaotic of two faces: A physical characters carrying on two faces of geometric tape graph like color, electricity, magnetic filed...etc.

1-The matrix representation of geometric tape graph

Considers the geometric graph $G\left(v^{0} v^{1}\right)$. See Fig. (1).

$<$ Figure $1>$

Its adjacent and incidence matrices are:

$A(G)=\left[\begin{array}{ll}0 & 1 \\ 1 & 0\end{array}\right]_{2}, I(G)=\left[\begin{array}{l}1 \\ 1\end{array}\right]_{2}$.

2- The matrix representation of chaotic tape graph

Considers the chaotic graph $G_{h}\left(v_{0 h}^{0} v_{0 h}^{1}\right)$, this graph consists of the geometric edges $e_{0 h}^{1}$ and smooth chaotic edges $e_{i h}^{1}, i=$ $1,2,3, \ldots, \infty$, of one maximum point see Fig.(2).

The adjacent and incidence matrices of this chaotic tape graph are:

$A\left(G_{h}\right)=\left[\begin{array}{ll}0_{0123 \ldots \infty h} & 1_{0123 \ldots \infty h} \\ 1_{0123 \ldots \infty h} & 0_{0123 \ldots \infty h}\end{array}\right]_{2}, I\left(G_{h}\right)=\left[\begin{array}{l}1_{0123 \ldots \infty h} \\ 1_{0123 \ldots \infty h}\end{array}\right]_{2}$.

These matrcies represent the chaotic of one face.

$<$ Figure $2>$

Chaotic of loop tape graph:

A loop is an edge joining a vertex to itself. See Fig.(3), we can represent chaotic of loop tape graph see Fig.(4). The adjacent and incidence matrices are:

$A(G)=[1]_{2}, I(G)=[1]_{2}$

$A\left(G_{h}\right)=\left[1_{0123 \ldots \infty h}\right]_{2}, I\left(G_{h}\right)=\left[1_{0123 \ldots \infty h}\right]_{2}$

These matrcies represent the chaotic of one face.

$<$ Figure $3>$

$<$ Figure $4>$

Complete chaotic of tape graphs:

A simple geometric tape graph in which each pair of distinct vertices are adjacent is a complete tape graph. We denote the complete tape graph on $n$ vertices $k, k$ has $n(n-1) / 2$ edges see Fig.(5). 
$<$ Figure $5>$

And Its adjacent and incidence matrices are:

$A(G)=\left[\begin{array}{lll}0 & 1 & 1 \\ 1 & 0 & 1 \\ 1 & 1 & 0\end{array}\right]_{2}, I(G)=\left[\begin{array}{lll}1 & 0 & 1 \\ 1 & 1 & 0 \\ 0 & 1 & 1\end{array}\right]_{2}$

We can represent chaotic of complete tape graph see Fig.(6). And It's adjacent and incidence matrices are:

$A\left(G_{h}\right)=\left[\begin{array}{lll}0_{0123 \ldots \infty h} & 1_{0123 \ldots \infty h} & 1_{0123 \ldots \infty h} \\ 1_{0123 \ldots \infty h} & 0_{0123 \ldots \infty h} & 1_{0123 \ldots \infty h} \\ 1_{0123 \ldots \infty h} & 1_{0123 \ldots \infty h} & 0_{0123 \ldots \infty h}\end{array}\right]_{2}, I\left(G_{h}\right)=\left[\begin{array}{lll}1_{0123 \ldots \infty h} & 0_{0123 \ldots \infty h} & 1_{0123 \ldots \infty h} \\ 1_{0123 \ldots \infty h} & 1_{0123 \ldots \infty h} & 0_{0123 \ldots \infty h} \\ 0_{0123 \ldots \infty h} & 1_{0123 \ldots \infty h} & 1_{0123 \ldots \infty h}\end{array}\right]_{2}$.

$<$ Figure $6>$

To represent a chaotic of two faces and It's adjacent and incidence matrices are:

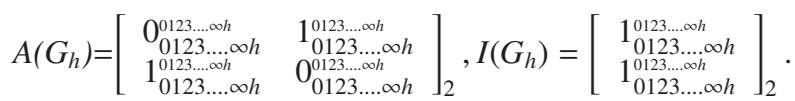

See Fig.(7).

$<$ Figure $7>$

And its loop represented by adjacent and incidence matrices:

$A\left(G_{h}\right)=\left[1_{0123 \ldots \infty}^{0123 \ldots \infty}\right]_{2}, I\left(G_{h}\right)=\left[1_{0123 \ldots \infty}^{0123 \ldots \infty}\right]_{2}$, see Fig.(8). Inside pure chaotic loops and outside chaotic loops.

$<$ Figure $8>$

The adjacent and incidence matrices of complete chaotic of two faces are:

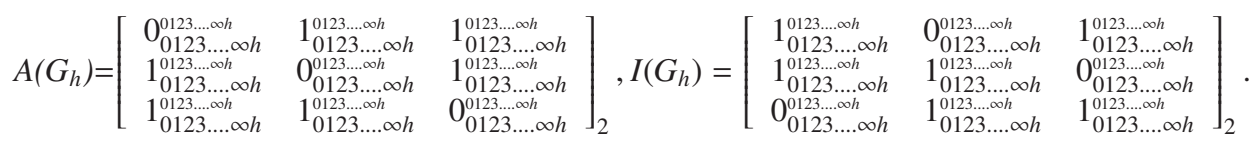

Tree tape graphs:

A tree tape graph is connected tape graph with only one path between each pair of vertices containing no cycles see Fig. (9).

\section{$<$ Figure $9>$}

It's adjacent and incidence matrices are:

$A(G)=\left[\begin{array}{lllllll}0 & 1 & 0 & 0 & 0 & 0 & 0 \\ 1 & 0 & 1 & 1 & 1 & 0 & 0 \\ 0 & 1 & 0 & 0 & 0 & 1 & 0 \\ 0 & 1 & 0 & 0 & 0 & 0 & 1 \\ 0 & 1 & 0 & 0 & 0 & 0 & 0 \\ 0 & 0 & 1 & 0 & 0 & 0 & 0 \\ 0 & 0 & 0 & 1 & 0 & 0 & 0\end{array}\right]_{2}, I(G)=\left[\begin{array}{llllll}1 & 0 & 0 & 0 & 0 & 0 \\ 1 & 1 & 1 & 1 & 0 & 0 \\ 0 & 1 & 0 & 0 & 1 & 0 \\ 0 & 0 & 1 & 0 & 0 & 1 \\ 0 & 0 & 0 & 1 & 0 & 0 \\ 0 & 0 & 0 & 0 & 1 & 0 \\ 0 & 0 & 0 & 0 & 0 & 1\end{array}\right]_{2}$

On chaotic tape tree graphs:

The adjacent and incidence matrices of one side are:

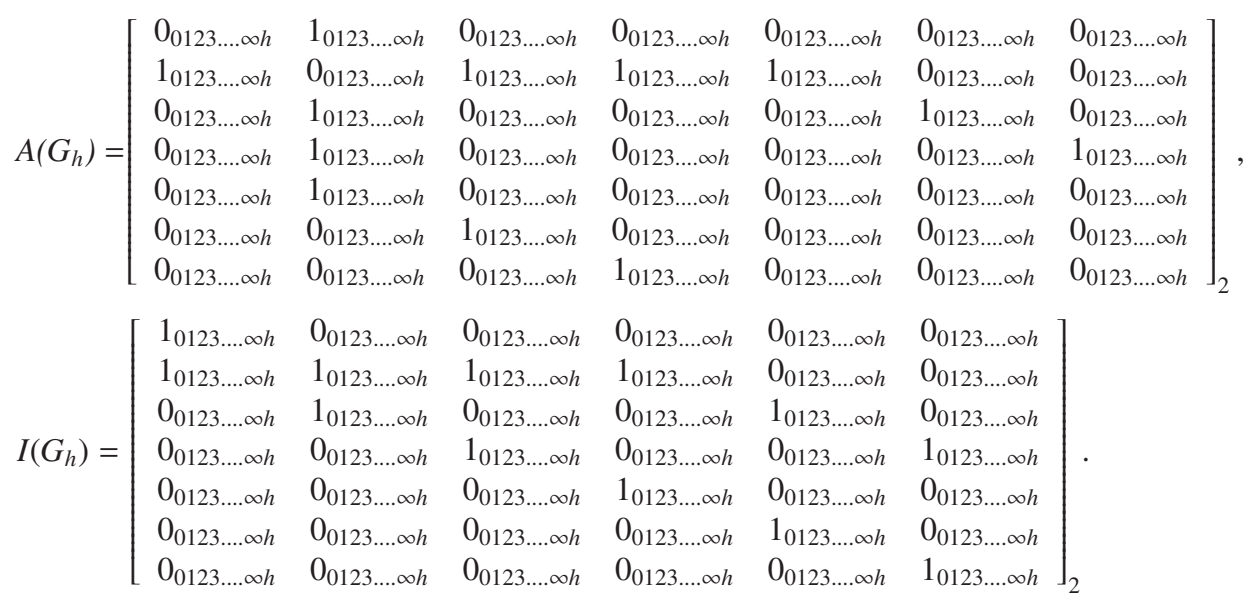


And Its adjacent and incidence matrices of two side are:

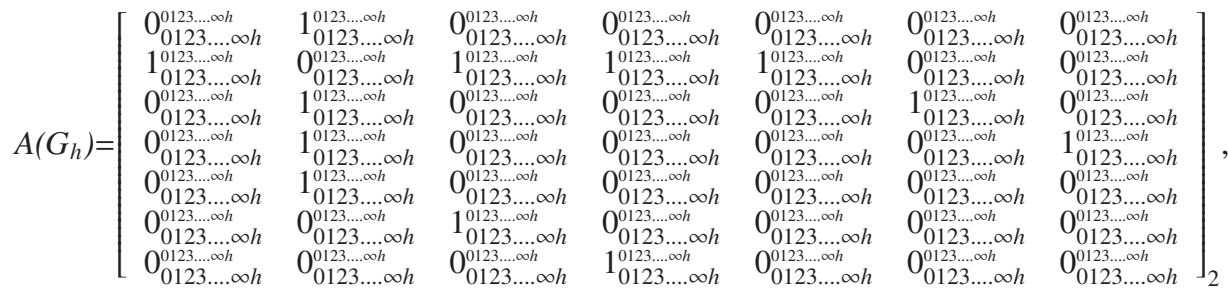

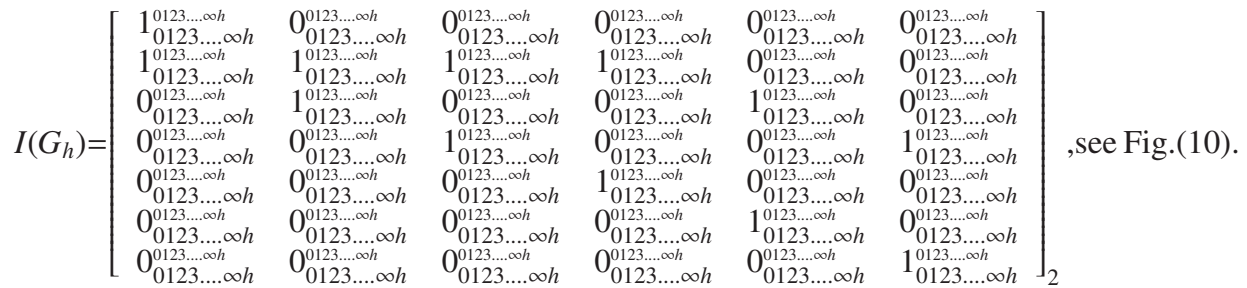

$<$ Figure $10>$

\subsection{Folding of chaotic tape graph}

There are two type of folding, we will illustrate their in the following theorems:

Theorem 2.2.1:

The folding of the geometric tape graph induce the same type of folding of all chaotics.

\section{Lemma 2.2.1:}

The limit of foldings of the geometric tape graph induce the limit of all foldings of chaotics.

\section{Proof:}

Consider the chaotic tape graph $G_{h}$ which consists of the geometric edge $e_{0 h}^{1}$ and the chaotic edges $e_{i h}^{1}, i=1,2, . . \infty$. Let $f: G_{h} \rightarrow G_{h}$ be a folding edge $e_{0 h}^{1}$ and the chaotic edges $e_{i h}^{1}$ such that $f_{1}: G_{h} \rightarrow G_{h 1}, f_{2}: G_{h 1} \rightarrow G_{h 2}, f_{3}: G_{h 2} \rightarrow$ $G_{h 3}, \ldots . .,{ }_{n} \lim _{\infty} f_{n}\left(G_{h n-1}\right)=G_{h n}$ which is the usual graph see Fig.(11).

$<$ Figure $11>$

Also by matrix:

$$
\begin{aligned}
& A\left(G_{h}\right)=\left[\begin{array}{ll}
0_{0123 \ldots \infty h} & 1_{0123 \ldots \infty h} \\
1_{0123 \ldots \infty h} & 0_{0123 \ldots \infty h}
\end{array}\right]_{2} \underset{f_{1}}{\rightarrow}\left[\begin{array}{ll}
0_{0123 \ldots \infty h} & 1_{0123 \ldots \infty h} \\
1_{0123 \ldots \infty h} & 0_{0123 \ldots \infty h}
\end{array}\right]_{2} \underset{f_{2}}{\rightarrow}\left[\begin{array}{ll}
0_{0123 \ldots \infty h} & 1_{0123 \ldots \infty h} \\
1_{0123 \ldots \infty h} & 0_{0123 \ldots \infty h}
\end{array}\right]_{2} \\
& \stackrel{\ldots . . . f_{k}}{\longrightarrow}\left[\begin{array}{cc}
0_{0123 \ldots \infty h} & 1_{0123 \ldots \infty h} \\
1_{0123 \ldots \infty h} & 0_{0123 \ldots \infty h}
\end{array}\right] \stackrel{\lim _{\infty} f_{n}}{\longrightarrow}\left[0_{0123 \ldots \infty h}\right]
\end{aligned}
$$

And for incidence:

$I\left(G_{h}\right)=\left[\begin{array}{l}1_{0123 \ldots \infty h} \\ 1_{0123 \ldots \infty h}\end{array}\right]_{2} \underset{f_{1}}{\rightarrow}\left[\begin{array}{l}1_{0123 \ldots \infty h} \\ 1_{0123 \ldots \infty h}\end{array}\right]_{2} \underset{f_{2}}{\rightarrow}\left[\begin{array}{l}1_{0123 \ldots \infty h} \\ 1_{0123 \ldots \infty h}\end{array}\right]_{2} \underset{f_{k}}{\rightarrow}\left[\begin{array}{l}1_{0123 \ldots \infty h} \\ 1_{0123 \ldots \infty h}\end{array}\right]_{n} \underset{n}{\lim _{\infty} f_{n} \Phi}$.

\section{Theorem 2.2.2:}

The folding for the pure chaotics edges of a chaotic tape graph into itself does not induce a folding for the geometric edge.

\section{Proof:}

Consider the chaotic tape graph $G_{h}$ which consists of the geometric edge $e_{0 h}^{1}$ and the chaotic edges $e_{i h}^{1}, i=1,2, . . \infty$. Let $f: G_{h} \rightarrow G_{h}$ be a folding edge such that $f\left(G_{h}\right)=\overline{G_{h}}$ and $f\left(e_{i h}^{1}\right)=e_{j h}^{1}$ where $i \neq j, j=1,2, . . \infty$ then at each folding the number of the chaotic edges is reduced by 1 i.e. $f\left(e_{12 . . . . . j . . \infty h}^{1}\right)=e_{12 . . j . . \infty h}^{1}$. By repeating this folding many times then the chaotic tape graph $G_{h}$ becomes a 1-chaotic on its geometric edge $e_{0 h}^{1}$, then this type of folding does not induce a folding for the geometric edge.

\section{Lemma 2.2.2:}

The limit of foldings for the pure chaotics edges of a chaotic tape graph into itself have not any effect on the geometric edge. 


\section{Proof:}

Let $f: G_{h} \rightarrow G_{h}$ be a folding edge such that $f\left(G_{h}\right)=\overline{G_{h}}$ and $f\left(e_{i h}^{1}\right)=\overline{e_{i h}^{1}}$ where $\overline{e_{i h}^{1}}$ is a different density from $e_{i h}^{1} \forall i$. This different may be in the length or in the physical characters (reduce or increase), all these types of folding for the pure chaotics edges have not any effect on the geometric edge i.e. $e_{0 h}^{1}$ still invariant see Fig.(12).

$<$ Figure $12>$

Also by matrix:

$$
\begin{aligned}
& A\left(G_{h}\right)=\left[\begin{array}{cc}
0_{012}^{012} & 1_{012}^{012} \\
1_{012}^{012} & 0_{012}^{012}
\end{array}\right]_{2} \underset{1}{\rightarrow}\left[\begin{array}{cc}
0_{012_{f}}^{012_{f}} & 1_{012_{f}}^{012_{f}} \\
1_{012_{f}}^{012_{f}} & 0_{012_{f}}^{012_{f}}
\end{array}\right]_{2} \underset{f_{2}}{\rightarrow}\left[\begin{array}{cc}
0_{012_{f}}^{012_{f}} & 1_{012_{f}}^{012_{f}} \\
1_{012_{f}}^{012_{f}} & 0_{012_{f}}^{012_{f}}
\end{array}\right]_{2} \underset{f_{3}}{\rightarrow}\left[\begin{array}{ccc}
0_{01}^{01} & 1_{01}^{01} \\
1_{01}^{01} & 0_{01}^{01}
\end{array}\right]_{2} \\
& \stackrel{\ldots . . . . f_{k}}{\longrightarrow}\left[\begin{array}{cc}
0_{01_{f}}^{01_{f}} & 1_{01_{f}}^{01_{f}} \\
1_{01_{f}}^{01_{f}} & 0_{01_{f}}^{01_{f}}
\end{array}\right]_{2} \stackrel{\cdots}{\longrightarrow} \stackrel{\lim _{\infty} f_{n}}{\longrightarrow}\left[\begin{array}{cc}
0_{01_{f}} & 1_{01_{f}} \\
1_{01_{f}} & 0_{01_{f}}
\end{array}\right]_{2}
\end{aligned}
$$

And for incidence:

$$
\begin{aligned}
I\left(G_{h}\right) & =\left[\begin{array}{c}
1_{012}^{012} \\
1_{012}^{012}
\end{array}\right]_{2} \underset{f_{1}}{\rightarrow}\left[\begin{array}{l}
1_{012_{f}}^{012_{f}} \\
1_{012_{f}}^{012_{f}}
\end{array}\right]_{2} \underset{f_{2}}{\rightarrow}\left[\begin{array}{c}
1_{012_{f}}^{012_{f}} \\
1_{012_{f}}^{012_{f}}
\end{array}\right]_{2} \underset{f_{3}}{\rightarrow}\left[\begin{array}{c}
1_{01}^{01} \\
1_{01}^{01}
\end{array}\right]_{2} \underset{f_{k}}{\rightarrow}\left[\begin{array}{c}
1_{01_{f}}^{01_{f}} \\
1_{01_{f}}^{01_{f}}
\end{array}\right]_{2} \ldots . . \\
& \stackrel{\lim _{\infty} f_{n}}{\longrightarrow}\left[\begin{array}{c}
1_{01_{f}} \\
1_{01_{f}}
\end{array}\right]_{2} .
\end{aligned}
$$

Where $1_{012_{f}}^{012_{f}}$ means that we make a folding for the $e_{2 h}^{1}$ chaotic edge.

\section{Lemma 2.2.3:}

The unfolding of the chaotics edges of tape graph into itself decreases the chaotics dgree.

\subsection{Retraction of chaotic tape graph}

There are two types of retractions for chaotics tape graph:

(a) Geometric retraction:

Making a retraction $r_{i}\left(G_{h}-\left\{v_{0 h}^{i}\right\}\right)=\left\{\begin{array}{c}v_{0 h}^{0}, i=1 \\ v_{0 h}^{1}, i=0\end{array}\right\} \rightarrow G_{h}^{1}$ for the geometric edge as we mentioned, consequently the chaotic edges will be retracted as the geometric edge, then we get a chaotic vertex at $v_{0 h}^{0}$ or $v_{0 h}^{1}$, the adjacent and the incidence matrices are:

$A\left(G_{h}^{1}\right)=\left[0_{0123 \ldots \infty h}\right]_{2}, I\left(G_{h}^{1}\right)=\Phi$.

Also by the retraction $r: G_{h}-\left\{e_{0 h}^{1}\right\} \rightarrow G_{h}^{2}$ we get two chaotic vertices $v_{0 h}^{0}$ and $v_{0 h}^{1}$, see Fig.(13) the adjacent and the incidence matrices are:

$A\left(G_{h}^{2}\right)=\left[\begin{array}{ll}0_{0123 \ldots \infty h} & 0_{0123 \ldots \infty h} \\ 0_{0123 \ldots \infty h} & 0_{0123 \ldots \infty h}\end{array}\right]_{2}, I\left(G_{h}^{2}\right)=\Phi$.

$<$ Figure $13>$

(b) Retraction of the density of the physical character:

Making a retraction for the chaotic edges $e_{i h}^{1}, i=1,2, \ldots \infty h$ of the chaotic graph $G_{h}$, by removing an interior point which leads to reduce the density of the physical character or vanishing the chaotic edge which we make a retraction for it, and that has no effect on the geometric edge or on the other chaotic edges. So by the retraction

$r: G_{h}-\left\{e_{i h}^{1}\right\} \rightarrow G_{h}^{3}$,we can reduce the density of the chaotic edges.

The adjacent and the incidence matrices are:

$A\left(G_{h}\right)=\left[\begin{array}{ll}0_{0123 \ldots \infty h} & 1_{01} 2 \ldots \infty h \\ 1_{01_{r} \ldots \ldots \infty} & 0_{0123 \ldots \infty h}\end{array}\right]_{2}, I\left(G_{h}\right)=\left[\begin{array}{c}1_{01_{r} 2 \ldots \infty h} \\ 1_{01_{r} 2 \ldots \infty h}\end{array}\right]_{2}$.

Where $1_{01_{r} 2 . . \infty h}$ means that we make a retraction for the chaotic edge $e_{1 h}^{1}$ this retraction is equivalent to the folding of the chaotic edge $e_{1 h}^{1}$ on $e_{2 h}^{1}$ and that has no effect on the geometric edge or the other chaotic edges.

\section{Theorem 2.3.1:}

Any type of retractions for the geometric tape graph of a chaotic tape graph into itself induces the same retraction for chaotic edges.

\section{proof:}


Let $r: G_{h}-\{v\} \rightarrow \overline{G_{h}}$ be a retraction for the geometric edge of the chaotic tape graph $G_{h}, v$ is either a vertex $\left(v_{0 h}^{0}\right.$ or $v_{1 h}^{0}$ ) or an interior point $v_{0 h}$ on this edge.

So we get the following retraction's cases:

1- $r^{1}: G_{h}-\left\{v_{0 h}^{0}\right\} \rightarrow \overline{G_{h}}$ is define as $r^{1}\left(e_{0 h}^{1}-\left\{v_{0 h}^{0}\right\}\right)=v_{0 h}^{1}$, then there is an induced sequence of retractions of it's chaotic $r_{i}^{1}: G_{h}-\left\{v_{i h}^{0}\right\} \rightarrow \overline{G_{h}}, i=1,2, . . \infty$, such that:

- $r_{1}^{1}\left(e_{1 h}^{1}-\left\{v_{1 h}^{0}\right\}\right)=v_{1 h}^{1}$,

- $r_{2}^{1}\left(e_{2 h}^{1}-\left\{v_{2 h}^{0}\right\}\right)=v_{2 h}^{1}, \ldots$.

- $r_{\infty}^{1}\left(e_{\infty h}^{1}-\left\{v_{\infty h}^{0}\right\}\right)=v_{\infty h}^{1}$.

2- $r^{2}: G_{h}-\left\{v_{0 h}^{0}\right\} \rightarrow \overline{\overline{G_{h}}}$ is defined as $r^{2}\left(e_{0 h}^{1}-\left\{v_{0 h}^{1}\right\}\right)=v_{0 h}^{0}$, then there is an induced sequence of retractions of it's chaotic $r_{i}^{2}: G_{h}-\left\{v_{i h}^{0}\right\} \rightarrow \overline{\overline{G_{h}}}, i=1,2, . . \infty$,such that:

- $r_{1}^{2}\left(e_{1 h}^{1}-\left\{v_{1 h}^{1}\right\}\right)=v_{1 h}^{0}$,

- $r_{2}^{2}\left(e_{2 h}^{1}-\left\{v_{2 h}^{1}\right\}\right)=v_{2 h}^{0}, \ldots .$.

- $r_{\infty}^{2}\left(e_{\infty h}^{1}-\left\{v_{\infty h}^{1}\right\}\right)=v_{\infty h}^{0}$.

3- $r^{3}: G_{h}-\left\{v_{0 h}\right\} \rightarrow \overline{\overline{G_{h}}}, v_{0 h} \neq v_{0 h}^{0}, v_{0 h} \neq v_{1 h}^{0}$, is define as $r^{3}\left(e_{0 h}^{1}-\left\{v_{0 h}\right\}\right)=\left\{v_{0 h}^{0}, v_{0 h}^{1}\right\}$ then there is an induced sequence of retractions of it's chaotic $r_{i}^{3}: G_{h}-\left\{v_{i h}\right\} \rightarrow \overline{\overline{G_{h}}}, i=1,2, . . \infty$,such that:

- $r_{1}^{3}\left(e_{1 h}^{1}-\left\{v_{1 h}\right\}\right)=\left\{v_{1 h}^{0}, v_{1 h}^{1}\right\}$,

- $r_{2}^{3}\left(e_{2 h}^{1}-\left\{v_{2 h}\right\}\right)=\left\{v_{2 h}^{0}, v_{2 h}^{1}\right\}, \ldots$,

- $r_{\infty}^{3}\left(e_{\infty h}^{1}-\left\{v_{\infty h}\right\}\right)=\left\{v_{\infty h}^{0}, v_{\infty h}^{1}\right\}$.

Theorem 2.3.2:

Any type of retractions for the pure chaotic system of a chaotic tape graph into itself does not induce any retraction for the geometric edge.

proof:

Let $r_{i}: G_{h}-\left\{v_{i h}\right\} \rightarrow \overline{G_{h}}$ be a retraction for the pure chaotic edges of the chaotic tape graph $G_{h}, v_{i h}$ is either a chaotic vertex $\left(v_{i h}^{0}\right.$ or $\left.v_{i h}^{0}\right)$ or an interior chaotic point $v_{i h}$ on the chaotic edges. So we get the following retraction's cases:

1- $r_{i}^{1}: G_{h}-\left\{v_{i h}^{0}\right\} \rightarrow \overline{G_{h}}, i=1,2, . . \infty$ are defined as $r_{i}^{1}\left(e_{i h}^{1}-\left\{v_{i h}^{0}\right\}\right)=v_{i h}^{1}$, then there are induced sequences of retractions of their chaotics such that:

- $r_{1}^{1}\left(e_{1 h}^{1}-\left\{v_{1 h}^{0}\right\}\right)=v_{1 h}^{1}$,

- $r_{2}^{1}\left(e_{2 h}^{1}-\left\{v_{2 h}^{0}\right\}\right)=v_{2 h}^{1}, \ldots$.

- $r_{\infty}^{1}\left(e_{\infty h}^{1}-\left\{v_{\infty h}^{0}\right\}\right)=v_{\infty h}^{1}$.

These retraction's cases do not induce a retraction for the geometric edge i.e. $r_{i}^{1}:\left(e_{i h}^{1}-\left\{v_{i h}^{0}\right\}\right) \nRightarrow r^{1}\left(e_{0 h}^{1}-\left\{v_{0 h}^{0}\right\}\right)$

2- $r_{i}^{2}: G_{h}-\left\{v_{i h}^{0}\right\} \rightarrow \overline{\overline{G_{h}}} i=1,2, . . \infty$ are defined as $r^{2}\left(e_{i h}^{1}-\left\{v_{i h}^{1}\right\}\right)=v_{i h}^{0}$, then there are induced sequences of retractions of their chaotics such that:

- $r_{1}^{2}\left(e_{1 h}^{1}-\left\{v_{1 h}^{1}\right\}\right)=v_{1 h}^{0}$,

- $r_{2}^{2}\left(e_{2 h}^{1}-\left\{v_{2 h}^{1}\right\}\right)=v_{2 h}^{0}, \ldots$.

- $r_{\infty}^{2}\left(e_{\infty h}^{1}-\left\{v_{\infty h}^{1}\right\}\right)=v_{\infty h}^{0}$.

These retraction's cases do not induce a retraction for the geometric edge i.e. $r_{i}^{2}:\left(e_{i h}^{1}-\left\{v_{i h}^{1}\right\}\right) \nRightarrow r^{2}\left(e_{0 h}^{1}-\left\{v_{0 h}^{1}\right\}\right)$.

3- $r_{i}^{3}: G_{h}-\left\{v_{i h}\right\} \rightarrow \overline{\overline{G_{h}}}, v_{i h} \neq v_{i h}^{0}, v_{i h} \neq v_{i h}^{0}, i=1,2, . . \infty$ are defined as $r_{i}^{3}\left(e_{i h}^{1}-\left\{v_{i h}\right\}\right)=\left\{v_{i h}^{0}, v_{i h}^{1}\right\}$ then there are the induced sequences of retractions of their chaotics such that:

- $r_{1}^{3}\left(e_{1 h}^{1}-\left\{v_{1 h}\right\}\right)=\left\{v_{1 h}^{0}, v_{1 h}^{1}\right\}$, 
- $r_{2}^{3}\left(e_{2 h}^{1}-\left\{v_{2 h}\right\}\right)=\left\{v_{2 h}^{0}, v_{2 h}^{1}\right\}, \ldots$,

- $r_{\infty}^{3}\left(e_{\infty h}^{1}-\left\{v_{\infty h}\right\}\right)=\left\{v_{\infty h}^{0}, v_{\infty h}^{1}\right\}$.

These retraction's cases do not induce a retraction for the geometric edge i.e. $r_{i}^{3}:\left(e_{i h}^{1}-\left\{v_{i h}\right\}\right) \nRightarrow r^{3}\left(e_{0 h}^{1}-\left\{v_{0 h}\right\}\right)$.

Lemma 2.3.1:

Any retraction for some chaotic edges, doesn't induces a retraction for the remaining chaotic edges.

See Fig.(14).

$<$ Figure $14>$

\subsection{Deformation of chaotic tape graph}

Definition 2.4.1: The deformation of geometric tape graph is the change in the metric properties of a continuous tape graph $G$ in the displacement from an initial placement $G_{0}$ to a final placement $G$. A change in the metric properties means that the edges and vertices drawn in the initial tape graph placement changes its length of edges and vertices when displaced to a tape graph in the final placement. So the deformation is a change in the shape or size of a tape graph due to an applied force.

\section{Theorem 2.4.1:}

Any type of deformation for the geometric tape graph of a chaotic tape graph into itself induces the same deformation for chaotic edges.

\section{proof:}

Consider the chaotic tape graph $G_{h}$ which consists of the geometric edge $e_{0 h}^{1}$ and the chaotic edges $e_{i h}^{1}, i=1,2, . . \infty$. Let $d: G_{h} \rightarrow \overline{G_{h}}$ be a deforming edge such that $e_{0 h}^{1}$ and the chaotic edges $e_{i h}^{1}$ such that $d_{1}: G_{h} \rightarrow G_{h 1}, d_{2}: G_{h 1} \rightarrow G_{h 2}$, $d_{3}: G_{h 2} \rightarrow G_{h 3}, \ldots . .,{ }_{n} \lim _{\infty} d_{n}\left(G_{h n-1}\right)=G_{h n}$ which is a random graph,see Fig.(15).

$<$ Figure $15>$

\section{Lemma 2.4.1:}

The limit of sequances of deformations of a chaotic tape graph is one of these deformations.

\section{Theorem 2.4.2:}

Any type of deformation for the pure chaotic system of a chaotic tape graph into itself does not induce any deformation for the geometric edge.

\section{proof:}

Consider the chaotic tape graph $G_{h}$ which consists of the geometric edge $e_{0 h}^{1}$ and the chaotic edges $e_{i h}^{1}, i=1,2, . . \infty$. Let $d: G_{h} \rightarrow G_{h}$ be a deforming pure chaotic edges such that $d\left(G_{h}\right)=\overline{G_{h}}$ and $d\left(e_{i h}^{1}\right)=\overline{e_{i h}^{1}}$ where $\overline{e_{i h}^{1}}$ is a different form $e_{i h}^{1} \forall$ $i$. This different in the physical characters (reduce or increase), all these types of deforming for the pure chaotics edges have not any effect on the geometric edge i.e. $e_{0 h}^{1}$ still invariant, see Fig.(16).

$<$ Figure $16>$

\section{Applications:}

(1) A ribbon of cassette recorder its geometric tape graph and chaotic tape graph, it has physical characters (colors and voices).

(2) A ribbon of camera film its geometric tape graph and chaotic tape graph, it has physical characters (colors and reflection).

(3) A ribbon of cassette video recorder its geometric tape graph and chaotic tape graph, it has physical characters (colors, voices and dynamics).

(4) A magnetic shapes which we put it on a fridge.

(5) Changing voices or colors in the screen of the television.

(6) Crash a plane induces to jamming of wireless set of it.

(7) Jamming of wireless set does not induce any deformation to the set itself.

(8) The rainbow is a chaotic tape graph. 


\section{References}

El-Ghoul, M. (1993). Folding of fuzzy graphs and fuzzy spheres. Fuzzy sets and systems, 58, 355-363, North-Holland.

El-Ghoul, M. Unfolding of graph and uncertain graph. The Australian mathematics journal, sandy Bay 7006, Tasmania, Australia.

El-Ghoul, M., El-Ahmady, A. and Homoda, T. (2006). On chaotic graphs and applications in physics and biology. Chaos, Solitions and fractals, 27, 159-173, UK.

El-Ghoul, M, El-Ahmady, A. and Rafat, H. (2004). Folding-retraction of chaotic dynamical manifold and the VAK of vacuum fluctuation. chaos, Solutions and Fractals, UK, 20:209-217.

El-Ghoul, M, El-Zohny, H, Khalil, M, M. (2010). On tape graphs. Journal of mathematics research. Vol.2, No.4. Issn:1916-9795, Canada.

Gibbons, A. (1995). Algorithmic graph theory. Cambridge University Press, Cambridge, UK.

Giblin, P.J. (1977). Graphs, surfaces and homology, an introduction to algebraic topology. Chapman and Hall. Ltd, London.

Gross, J.L. and Tucker, T.W. (1987). Topological graph theory. Jon Wiley \& Sons, Inc, Canada1987.

Robertson, S.A. (1977). Isometric folding of Riemannian manifolds. Proc.Fac.Roy.Soc.Edinburgh, 77, $275-284$.

Wilson, R.J. (1972). Introduction to graph theory. Olivar\& Boyed, Edinburgh.

Wilson, R.J. and Watkins, J.J. (1990). Graphs, an introductory approach, a first course in discrete mathematics. Jon Wiley \& Sons Inc, Canada.

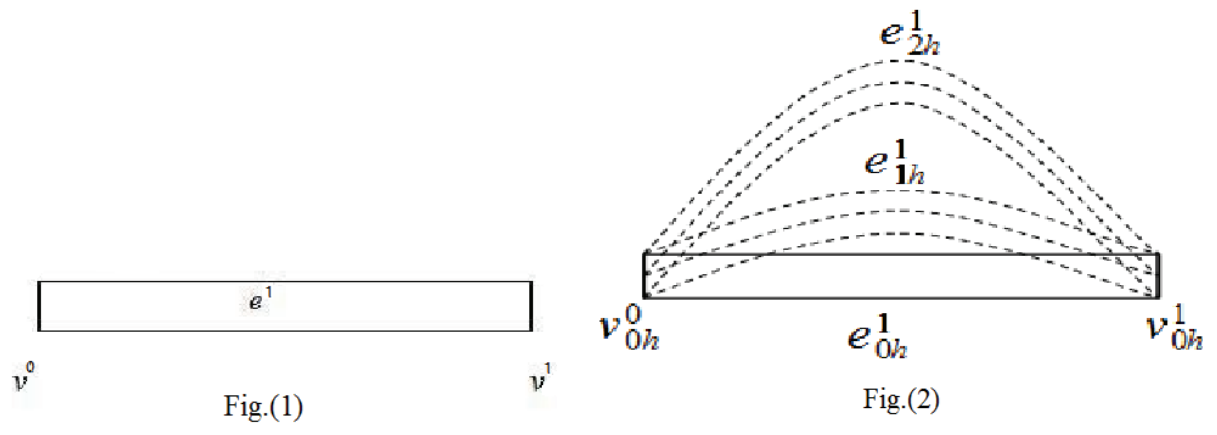

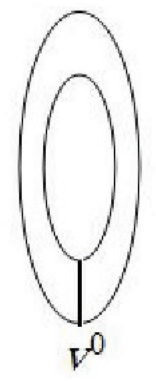

Fig.(3)

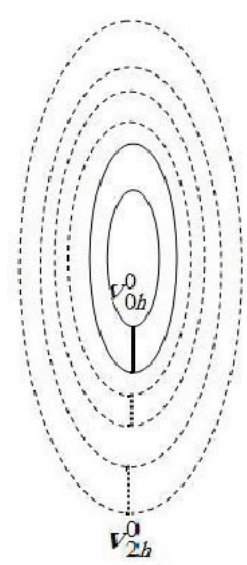

Fig.(4) 


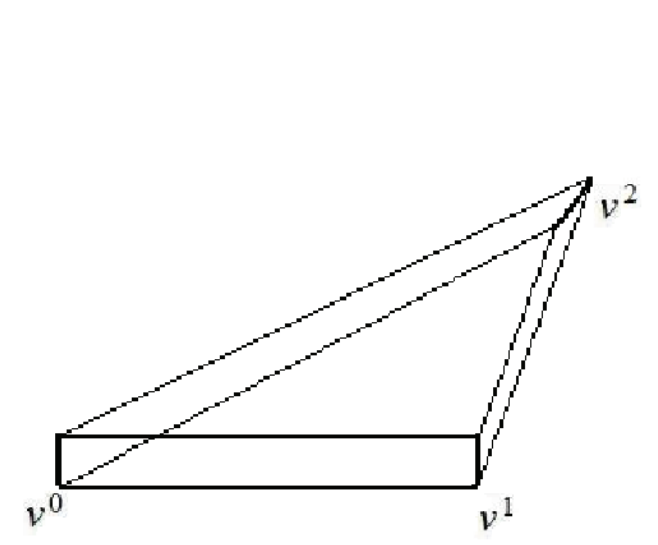

Fig.(5)

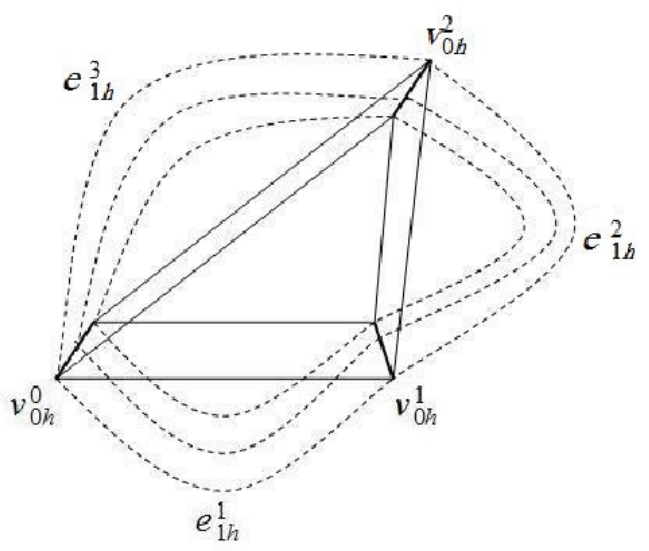

Fig.(6)

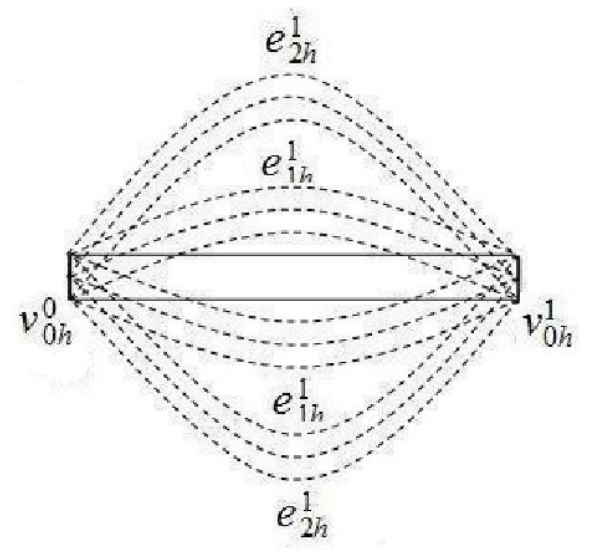

Fig.(7)

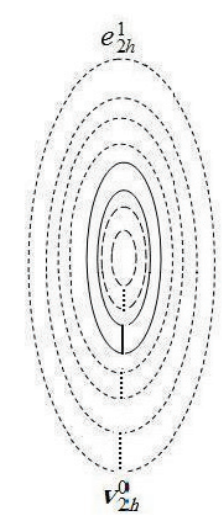

Fig.(8)

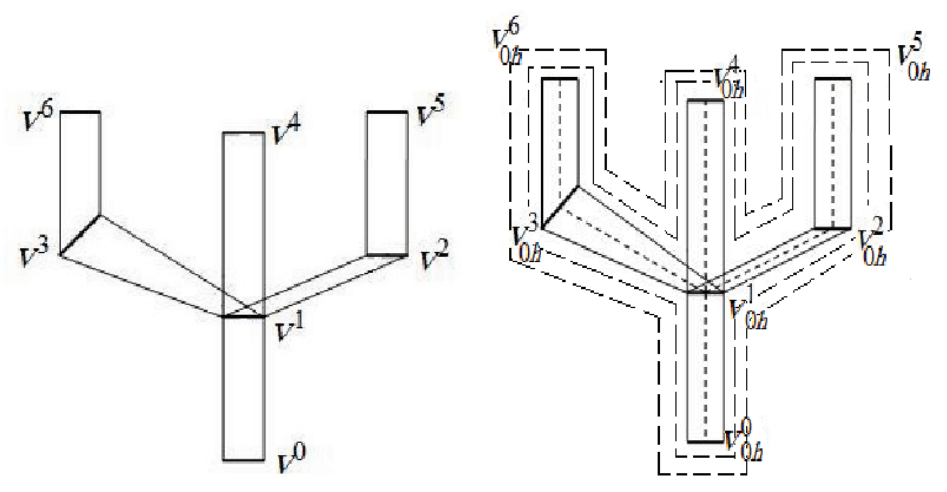

Fig.(9)

Fig.(10)

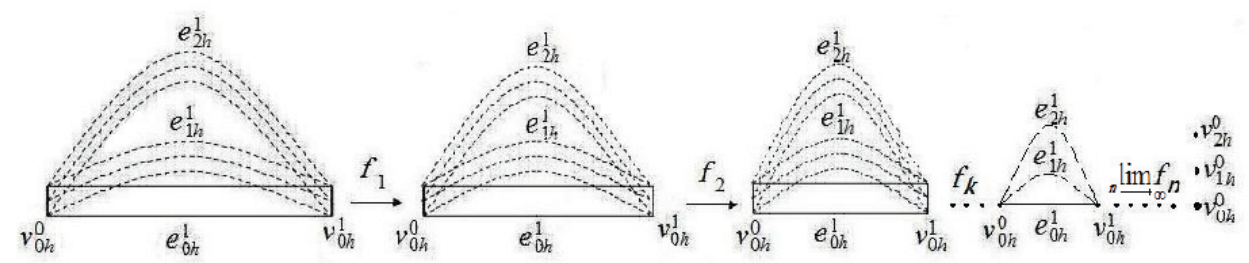

Fig.(11) 

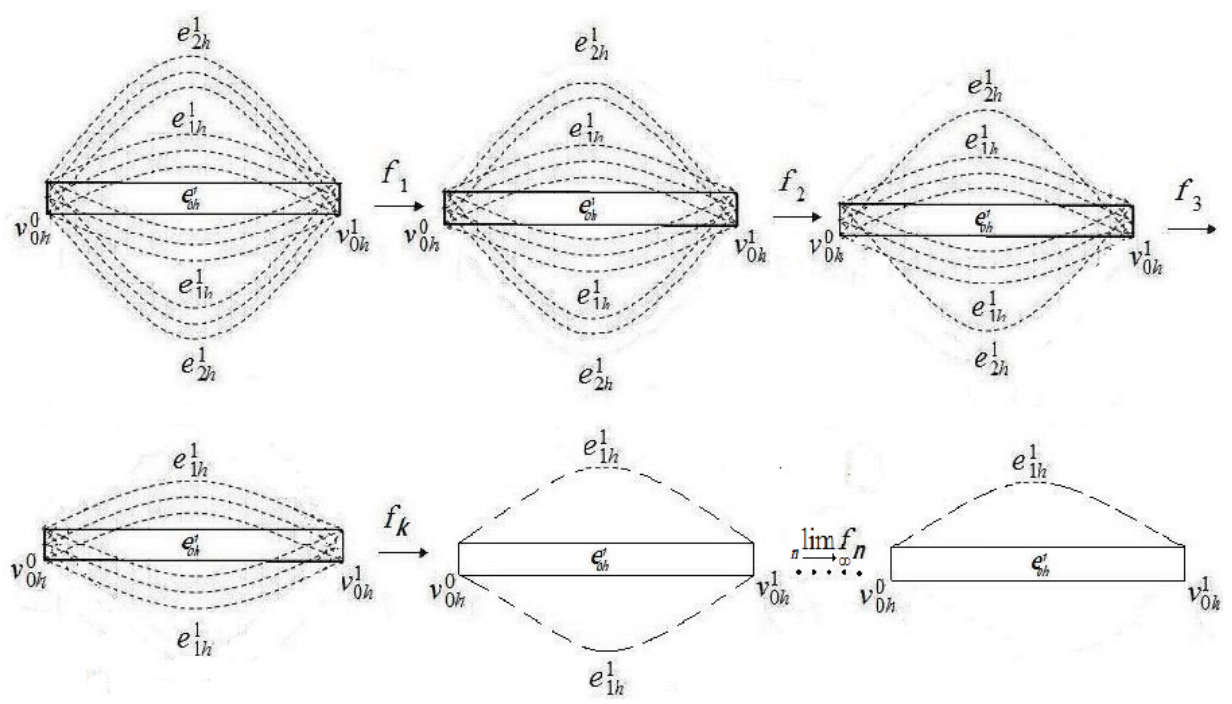

Fig.(12)

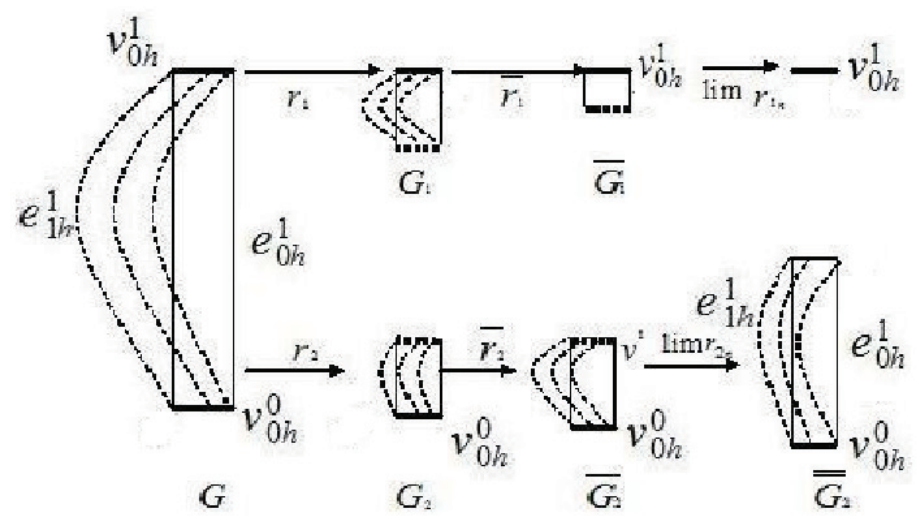

Fig.(13)
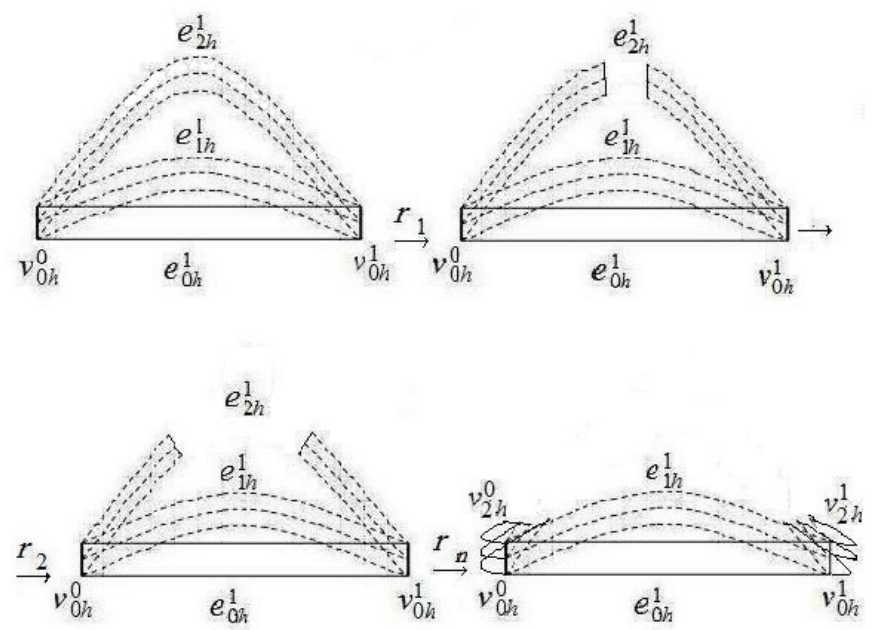

Fig.(14) 


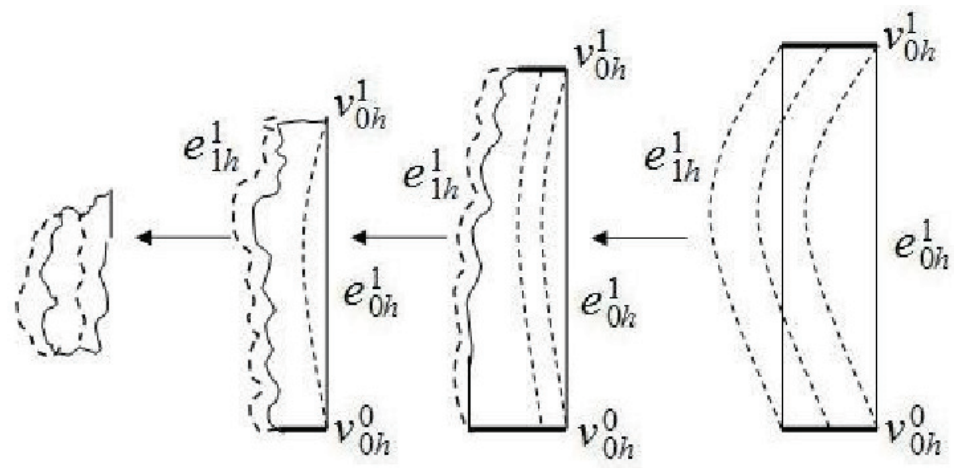

Fig.(15)
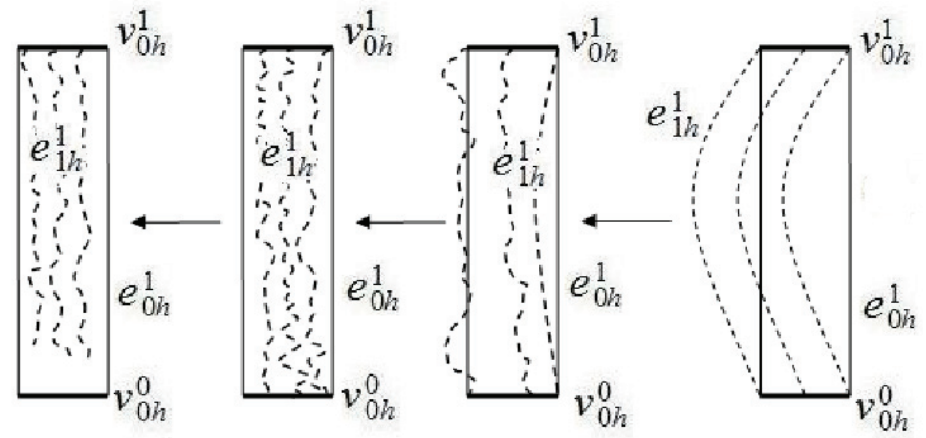

Fig.(16) 